\title{
Reducing inappropriate ESR testing with computerized clinical decision support
}

Stephanie Gottheil, Ekta Khemani, Katherine Copley, Michael Keeney, Jeff Kinney, lan Chin-Yee, Alan Gob

University of Western Ontario, Canada

\begin{abstract}
Laboratory test overutilization increases health care costs, leads to unwarranted investigations, and may have a negative impact on health outcomes. The American Society of Clinical Pathology, in its Choosing Wisely Campaign, advocates that inflammation be investigated with Creactive protein (CRP) instead of Erythrocyte Sedimentation Rate (ESR). London Health Sciences Centre (LHSC), a tertiary care hospital organization in Ontario, Canada, set a goal to reduce inappropriate ESR orders by $50 \%$. After developing appropriateness criteria for ESR, we used a series of PDSA cycles to reduce inappropriate ESR ordering and analyzed our results with an interrupted time series design. Our intervention began with an educational bulletin and moved to city-wide implementation of computerized Clinical Decision Support (CDS). After implementation, ESR orders decreased by $40 \%$ from 386 orders per week to 241 orders per week. Our results are supported by previous literature on the effectiveness of CDS in reducing overutilization and suggest that provider habit is a significant contributor to inappropriate ordering.
\end{abstract}

\section{Problem}

Unnecessary lab tests increase health care costs, lead to unwarranted investigations, and may negatively impact patient outcomes. The American Society of Clinical Pathology, in its Choosing Wisely Campaign, suggests inflammation be investigated with C-reactive protein (CRP) instead of Erythrocyte Sedimentation Rate (ESR).[1] London Health Sciences Centre (LHSC), a tertiarycare hospital organization in Ontario, Canada, targeted ESR for a pilot project to reduce test overutilization. LHSC is an urban, acutecare hospital with over one million patient visits per year. The division of Pathology and Laboratory Medicine (PaLM) runs over seven million laboratory tests annually with an operating budget of $\$ 55$ million CAD. The PaLM test utilization committee was created to develop strategies for reducing unnecessary laboratory testing. The team consisted of physicians and medical laboratory technologists, including the coordinator for hematology and the chair of the hematology division. The committee set a goal for this quality improvement (QI) initiative to reduce inappropriate ESR orders by $50 \%$.

\section{Background}

Appropriate laboratory test utilization is an important target of healthcare QI initiatives.[2] Some studies estimate that over $90 \%$ of laboratory tests could be considered unnecessary based on their ability to add value to patient care.[3] Not only does inappropriate test ordering increase healthcare costs, but it also may lead to additional unwarranted investigations that may have negative effects on patient outcomes.[2,4]

A recent systematic review compared the effectiveness of several interventions used to reduce laboratory test utilization.[2] The authors found that using multiple strategies, such as combining educational and systems-based interventions, was most successful. With the advent of electronic medical records and computerized provider order entry (CPOE), one focus of $\mathrm{QI}$ initiatives has been improving quality of care by using clinical decision support (CDS) to improve resource utilization.[5] CDS is an interactive information technology system that integrates evidence-based recommendations into the ordering process.[3] Roshanov et al.'s meta-analysis of computerized CDS interventions found that all four trials using CDS to reduce unnecessary testing were successful.[5]

The Choosing Wisely campaign was developed by the American Board of Internal Medicine to engage physicians and patients in identifying and decreasing unnecessary investigations.[6] Healthcare institutions have been using Choosing Wisely as a springboard for the development of their own test utilization initiatives.[6] LHSC chose ESR, a frequently overused test identified by the Choosing Wisely initiative, as a pilot project to explore strategies for effective test reduction.

ESR measures how quickly erythrocytes settle at the bottom of a vertical tube in a fixed time period. It is used as an indirect measure of inflammation, usually in conjunction with C-reactive protein (CRP), which is produced by the liver directly in response to inflammatory cytokines.[7] ESR is non-specific, elevated not only by inflammation but also by age, sex, anemia, pregnancy, low albumin, and kidney disease.[7] Moreover, a recent study showed that, in cases where ESR and CRP are discordant, at least $92 \%$ were due to "false-positive" ESR.[8] For these reasons, LHSC set a goal for this QI initiative to reduce inappropriate ESR orders by $50 \%$. This paper will describe the development and impact of a series of interventions aimed at changing ESR ordering practices.

\section{Baseline measurement}

LHSC uses Cerner Systems as it's electronic medical record, and 
implemented CPOE in 2014. In October 2014, the PaLM test utilization committee decided to focus on ESR as a pilot project to explore strategies for effective test reduction.

ESR has previously been targeted by laboratory organizations in New Zealand, Alberta, and British Columbia. An audit of all CPOE orders showed that at baseline (October 2014-October 2015), LHSC averaged 386 ESR orders per week and 580 CRP orders per week.

The PaLM committee developed a set of appropriateness criteria for ESR based on evidence from the research literature and the potential for patient impact. The criteria were discussed with stakeholders from services with the highest ordering frequencies (rheumatology, gastroenterology, orthopaedics, and emergency) either in person or through electronic mail (Table 1). We performed a baseline chart audit of 40 random ESR orders showing that $75 \%$ of ESR orders were inappropriate according to these criteria. Orders were selected using convenience sampling by a blinded medical laboratory technologist using a time-stamped list of all ESR orders between October 1 and December 31, 2014. The list included both inpatient and outpatient orders at all three hospital sites.

See supplementary file: ds7117.png - "ESR Appropriateness Criteria"

\section{Design}

We planned a series of interventions of increasing complexity, starting with an educational bulletin and moving to city-wide implementation of CDS. We used interrupted time series analysis to determine if our primary outcome, the number of ESR orders per week, was reduced by our interventions. Results were analyzed using statistical process control (SPC) charts (QI Macros and Microsoft Excel).

\section{Strategy}

PDSA Cycle 1: Educational Bulletin

The committee initially sent out an educational bulletin to all hospital staff via electronic mail in December 2014 advising that CRP is preferable to ESR and that these tests are rarely needed together. The one page bulletin identified guidelines for CRP and ESR ordering and cited the choosing wisely initiative. We hypothesized that this would reduce inappropriate ordering by targeting lack of provider of awareness.

\section{PDSA Cycle 2: Clinical Decision Support}

There is abundant literature to suggest that integrating CDS into CPOE can reduce unnecessary testing and improve quality of care.[4,5] Therefore, in addition to educating staff about evidencebased ESR ordering, the committee decided to trial computerized CDS based on the appropriateness criteria. A forcing function was included whereby the ESR order could not be entered without the clinician choosing an appropriate indication (Figure 1). Educational posters were distributed prior to implementation. We hypothesized that this would reduce inappropriate testing by targeting provider inertia due to habit, while also improving provider knowledge. The CDS tool was launched on October 20, 2015.

PDSA Cycle 3: Testing and Implementation

After CDS implementation, we encouraged feedback from clinicians. Many clinicians agreed that ESR was unnecessary after reviewing literature about their disease of interest. However, some clinicians required ESR for clinical research or scoring systems that were not included in the criteria. Furthermore, some clinicians felt that ESR should be allowed when clinical equipoise existed regarding the test's value, such as in SLE and other rheumatologic diseases.[21,22] Based on these discussions, we revised our CDS intervention and broadened ESR access to select subspecialties on November 10, 2015.

See supplementary file: ds7115.png - "Computerized CDS Tool"

\section{Results}

Our initial educational bulletin was sent on December 17, 2014 (PDSA Cycle 1) but did not have a significant impact on ESR orders. After CDS implementation on October 20, 2015 (PDSA Cycle 2), ESR orders per week decreased from 386 to 151 . When unlimited access was provided to select subspecialties on November 10, 2015 (PDSA Cycle 3) there was an increase in ESR orders per week to 241 . This represents a decrease of almost $40 \%$ from baseline (Figure 2), or a cost savings of roughly $\$ 11,000 \mathrm{CAD}$ per year.

After four weeks, we performed a chart audit of 40 random ESR orders to compare the indication provided by the ordering physician to the clinical situation. Orders were selected using convenience sampling by a blinded medical laboratory technologist given a timestamped list of all ESR orders between October 20 and December 31,2015 . The list included both inpatient and outpatient orders at all three hospital sites. We found that $40 \%$ of ESR orders did not match the indication given and would be considered inappropriate. Users with repeated inappropriate orders were contacted by electronic mail in order to reinforce our intervention.

See supplementary file: ds7116.pdf - "Figure 2"

\section{Lessons and limitations}

There were a number of valuable lessons learned during the QI process. Firstly, we discovered the importance of stakeholder engagement prior to the intervention and having decision leaders in each department to champion our cause. Departments that had support from decision leaders responded better to the initiative than those where this leadership was lacking.

Secondly, we learned the value of flexibility after implementation of any QI initiative; our ability to negotiate and work with stakeholders, 
as well as to deal with unexpected challenges, contributed greatly to the sustainability of this project. Lastly, we found that computerized CDS can be very effective, especially when combined with a "forcing function" through CPOE. While our initial educational intervention did not lead to significant changes, using CDS combined with education helped us to achieve our aim. This suggests that provider habit, as opposed to lack of knowledge, was the likely driver behind inappropriate test utilization.

Limitations of this project include a single centre design as well as a lack of appropriate balancing measures. While the CDS strategy was successful, it is difficult to know how many times this strategy could be implemented with other tests before creating excessive pop-up fatigue. Our chart audit after implementation showed that $40 \%$ of orders could still be considered inappropriate according to our criteria, which is an issue that we will need to target on an ongoing basis. As well, our solution to allow unfettered ESR access to a select number of subspecialties may be seen as unfair by other clinicians.

\section{Conclusion}

We present a report of a successful QI initiative to reduce inappropriate ESR testing using computerized CDS. Laboratory test overutilization is a major contributor to healthcare spending and can lead to negative patient outcomes. ESR was targeted as a frequently overused test consistent with Choosing Wisely guidelines. Our aim was to decrease testing by $50 \%$, and we were able to reduce the average number of ESR tests at our institution by $40 \%$. Our results are supported by previous literature on the effectiveness of CDS in reducing overutilization[3,4] and suggest that provider habit was a significant contributor to inappropriate ordering.

To ensure sustainability, ESR orders will be monitored with SPC charts in order to identify new special cause variation. Two members of the PaLM committee have been designated as process owners to monitor SPC charts and to use periodic audit-feedback for providers with recurrent inappropriate orders.

LHSC's electronic medical record, Cerner Systems, is used widely across North America. Therefore, this computerized CDS strategy could be used by other institutions to reduce ESR testing or could be adapted for other laboratory test utilization initiatives.

\section{References}

1 American Society of Clinical Pathology Recommendations [Internet]. Choosing Wisely Campaign. [Updated February 3, 2015] Available from: www.choosingwisely.org

2 Qaseem A, Alguire P, Dallas P, et al. Appropriate use of screening and diagnostic tests to foster high-value, cost-conscious care. Ann Intern Med 2012;156:147-9.

3 Kobewka DM, Ronksley PE, McKay JA, et al. Influence of educational, audit and feedback, system based, and incentive and penalty interventions to reduce laboratory test utilization: a systematic review. Clin Chem Lab Med 2015;53:157-83.

4 Roshanov PS, You JJ, Dhaliwal J, et al. Can computerized clinical decision support systems improve practitioners' diagnostic test ordering behavior? A decision-maker-researcher partnership systematic review. Implement Sci 2011;6:88.

5 Levick DL, Stern G, Meyerhoefer CD, et al. "Reducing unnecessary testing in a CPOE system through implementation of a targeted CDS intervention." BMC Med Inform Decis Mak 2013;13:43

6 Levinson W, Kallewaard M, Bhatia RS, et al. "Choosing Wisely": a growing international campaign. BMJ Qual Saf 2015;24:167-74.

7 Costenbader KH, Chibnik LB, Schur PH. Discordance between erythrocyte sedimentation rate and $\mathrm{C}$-reactive protein measurements: clinical significance. Clin Exp Rheumatol 2007;25:746-9.

8 Colombet I, Pouchot J, Kronz V, et al. Agreement between erythrocyte sedimentation rate and C-reactive protein in hospital practice. Am J Med 2010;123:863.e7-13.

9 Parikh M, Miller NR, Lee AG, et al. Prevalence of a normal Creactive protein with an elevated erythrocyte sedimentation rate in biopsy-proven giant cell arteritis. Ophthalmology 2006;113:1842-5.

10 Kermani TA, Schmidt J, Crowson CS, et al. Utility of erythrocyte sedimentation rate and C-reactive protein for the diagnosis of giant cell arteritis. Semin Arthritis Rheum 2012;41:866-71.

11 Cantini F, Salvarani C, Olivieri I, et al. Erythrocyte sedimentation rate and $\mathrm{C}$-reactive protein in the evaluation of disease activity and severity in polymyalgia rheumatica: a prospective follow-up study. Semin Arthritis Rheum 2000;30:17-24.

12 Greidanus NV, Masri BA, Garbuz DS, et al. Use of erythrocyte sedimentation rate and $\mathrm{C}$-reactive protein level to diagnose infection before revision total knee arthroplasty. A prospective evaluation. J Bone Joint Surg Am 2007;89:1409-16.

13 Ghanem E, Antoci V Jr, Pulido L, et al. The use of receiver operating characteristics analysis in determining erythrocyte sedimentation rate and C-reactive protein levels in diagnosing periprosthetic infection prior to revision total hip arthroplasty. Int J Infect Dis 2009;13:e444-9.

14 Michail M, Jude E, Liaskos C, et al. The performance of serum inflammatory markers for the diagnosis and follow-up of patients with osteomyelitis. Int J Low Extrem Wounds 2013;12:94-9.

15 van Asten SAV, Peters EJG, Xi Y, et al. The Role of Biomarkers to Diagnose Diabetic Foot Osteomyelitis. A Meta-analysis. Curr Diabetes Rev 2015; [Epub ahead of print].

16 Henry-Amar M, Friedman S, Hayat M, et al. Erythrocyte 
sedimentation rate predicts early relapse and survival in early-stage Hodgkin disease. The EORTC Lymphoma Cooperative Group. Ann Intern Med 1991;114:361-5.

17 Talebi-Taher M, Babazadeh S, Barati M, et al. Serum inflammatory markers in the elderly: are they useful in differentiating sepsis from SIRS? Acta Med Iran 2014;52:438-42.

18 Menees SB, Powell C, Kurlander J, et al. A meta-analysis of the utility of $\mathrm{C}$-reactive protein, erythrocyte sedimentation rate, fecal calprotectin, and fecal lactoferrin to exclude inflammatory bowel disease in adults with IBS. Am J Gastroenterol 2015;110:444-54.

19 Ernst AA, Weiss SJ, Tracy L-A, et al. Usefulness of CRP and ESR in predicting septic joints. South Med J 2010;103:522-6.

20 Baruah MP, Bhattacharya B. Significant role of serum CRP in differentiating inflammatory from non-inflammatory causes of thyrotoxicosis. Indian J Endocrinol Metab 2012;16:976-81.

21 Keenan RT, Swearingen CJ, Yazici Y. Erythrocyte sedimentation rate and C-reactive protein levels are poorly correlated with clinical measures of disease activity in rheumatoid arthritis, systemic lupus erythematosus and osteoarthritis patients. Clin Exp Rheumatol 2008;26:814-9.

22 Firooz N, Albert DA, Wallace DJ, et al. High-sensitivity Creactive protein and erythrocyte sedimentation rate in systemic lupus erythematosus. Lupus 2011;20:588-97.

23 Turner D, Mack DR, Hyams J, et al. C-reactive protein (CRP), erythrocyte sedimentation rate (ESR) or both? A systematic evaluation in pediatric ulcerative colitis. J Crohns Colitis 2011;5:423-9.

\section{Declaration of interests}

Nothing to declare.

\section{Acknowledgements}

None.

\section{Ethical approval}

Research ethics board approval was not sought as we felt our study met criteria for exemption based on the Tri-Council Policy Statement, Article 1.1 stating that quality assurance studies should be exempt from review. 